\title{
EFFICIENT AND RELIABLE PERFORMANCE OF A GOAL QUESTION METRICS APPROACH FOR RE-ENGINEERING SMALL SCALE TRANSACTION
}

\author{
S.ArunBalachandren ${ }^{1}$ \\ S. Raj Anand ${ }^{2}$ \\ ${ }^{1,2}$ Department of Computer Application \\ Vel Tech Multi Tech Dr. Rangarajan Dr. Sakunthala Engg. College, Chennai-600 065, \\ Tamil Nadu, India.
}

\begin{abstract}
Some of the literature survey have been made on the small scale transaction, only few of the transactions are build on Enterprise Resource Planning and till dated there is not such a methodology or an approach implemented on the small scale transaction. Several implementations are mainly focus on the large scale transaction and hence they are handles huge business volume. This paper proposed an approach for reengineering a small scale transaction by implementing GQM approach. Even though, web technology is most popular and reliable but these paper prove that re-engineering of small scale transaction on standalone application will be effective and reliable than web technology.
\end{abstract}

Keywords: GQM approach, small scale transaction, re-engineering,

\section{INTRODUCTION}

Implementing Approach: The Goal Question Metrics is a mechanism which providing a framework for the development of metrics program. The brief note of these approach are demonstrated on the $[4,10]$ which is used for the task characterization, construction, analysis, feedback and learning. The GQM approach is resulted and progress for many year practical experience.

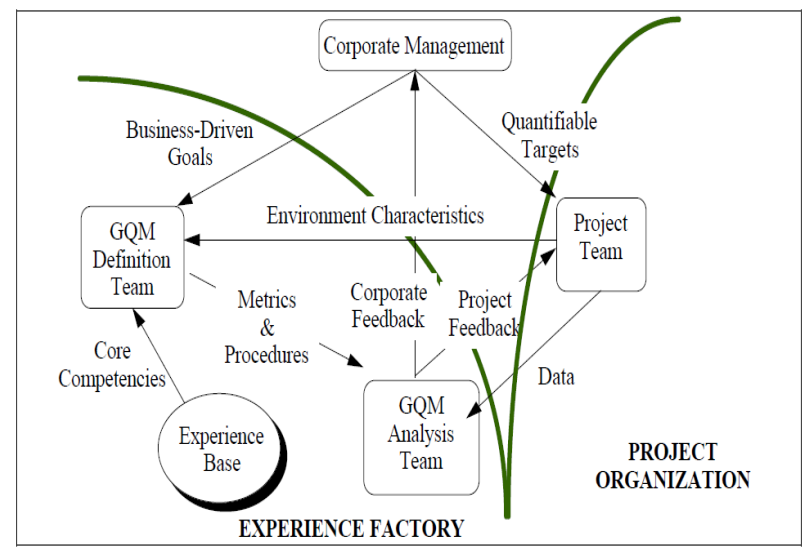

Fig 1: illustrates Goal Question Metrics working methodology

DOI : $10.14810 /$ ecij.2014.3102 
The formation of GQM approach is made in the form of corporate management as the higher official, who demand for their requirements. They forward their requirement to the GQM Definition team and to project team. The project development teams process the software engineering approach and forward their environment characteristics to GQM Definition Team.

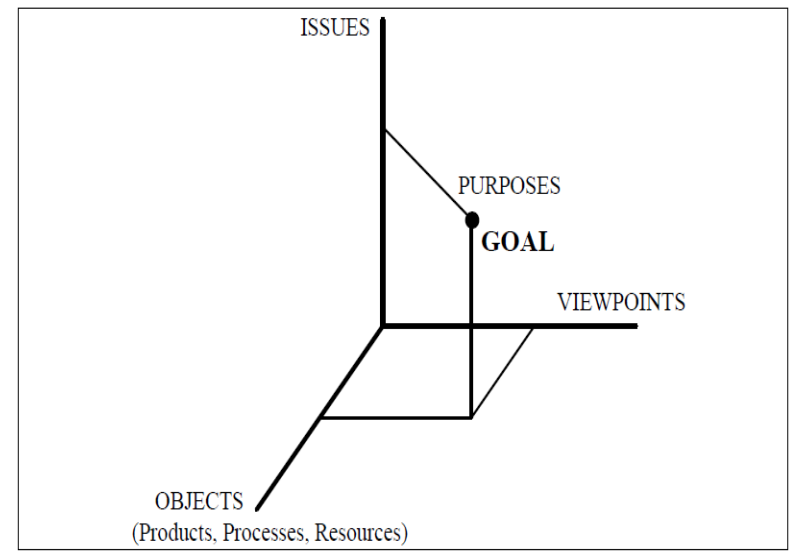

Fig 2: illustrates GQM activities

The figure 2 illustrates the triangular way of products, process, and resources to achieve the purpose as Goal. And then GQM Definition team analysis the question with the experience experts and make the metrics for each questions. After the formation of question and metrics, it forward to the GQM Analysis team. Now the GQM analysis team forwards the project feedback and receives the data from the project team.

In the GQM approach, definition phase is the second phase and mostly concentrates on all the activities.

Goal Measurement: This measurement is the mechanism of understanding the way and clear structure of the project development.

Question: This mean by the interpreted measurement of goals. By the way of answer these question, mean to conclude that the measurement goal will provide a new approach and it consider as a metrics or solution.

Metrics: is the method of number of solution which is suitable for more than one solution for a question and they are providing an analytic approach for the approach. 
Electrical \& Computer Engineering: An International Journal (ECIJ) Volume 3, Number 1, March 2014

\begin{tabular}{|c|c|c|}
\hline Goal & $\begin{array}{l}\text { Purpose } \\
\text { Issue } \\
\text { Object (process) } \\
\text { Viewpoint } \\
\end{array}$ & $\begin{array}{l}\text { Improve } \\
\text { the timeliness of } \\
\text { change request processing } \\
\text { from the project manager's viewpoint }\end{array}$ \\
\hline Question & & $\begin{array}{l}\text { What is the current change request processing } \\
\text { speed? }\end{array}$ \\
\hline Metrics & & $\begin{array}{l}\text { Average cycle time } \\
\text { Standard deviation } \\
\% \text { cases outside of the upper limit }\end{array}$ \\
\hline Question & & Is the performance of the process improving? \\
\hline Metrics & & 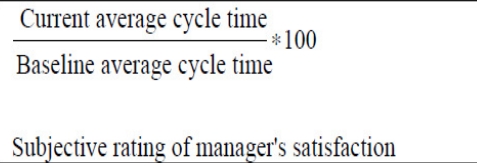 \\
\hline
\end{tabular}

Fig 3: illustrates sample GQM questions and its metrics

The figure 3 illustrates the sample Goal, Question and its metrics for project management and brief demonstrated [9].

\subsection{OTHER RELATED APPROACH [7]:}

Game Theory [3]: is one of the interactive decisions making approach which is mainly used by the decision maker at the time of emergency situation. It deals with the situation which consist of two or more actors where inter related (i.e.,) the outcomes is not depend on single player.

State B:

Cooperate Defect

\begin{tabular}{|c|c|c|c|}
\hline State A: & Cooperate & $\begin{array}{l}\text { Compromise } \\
(3.3)\end{array}$ & $\begin{array}{l}B \text { wins } \\
(2,4)^{*}\end{array}$ \\
\hline & Defect & $\begin{array}{l}\text { A wins } \\
(4,2)^{*}\end{array}$ & $\begin{array}{l}\text { Conflict } \\
(1,1)\end{array}$ \\
\hline
\end{tabular}

Key: $(\mathrm{x}, \mathrm{y})=$ payoff to State A, payoff to State B

4 = best; 3 = next-best; 2 = next-worst; $1=$ worst

$*$ = Pure strategy Nash equilibrium

Fig 4: illustrates Game Theory another approach

The figure 4 make a comparison of two state as State A and B. which consist of Cooperate and its defects. And is results the using game theory, which consists of 4 best, 3 of next best, 2 of next worst and 1 worst.

Valuation approach [1]: is performing major role in finance management. It is study of market efficiency and also question about the corporate organization which make a comparison between various decision rules. 


\subsection{PROJECT MANAGEMENT USING SDLC}

Hence, the Software Development is based on Enterprise Resource planning Package which performed on the combination of SDLC and Project Management [5]. The Software Development Lifecycle is consist of following figure as

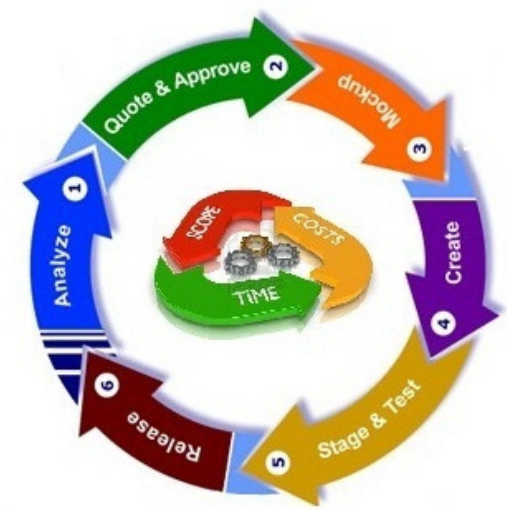

Fig 5: illustrates SDLC and Project Management

Analyze: deals with the basic requirement of project development

Quote \& Approve: hence project development based on GQM approve, each question and metrics are to be approved by the GQM definition team.

Mockup: This illustrates the structure of the project and experimental method.

Create: which maintain by project team

Stage \& Test: This is monitored by corporate organization and GQM analysis team

Release: This is said to be final phase of the SDLC.

Project Management: This is consist of triangular dependency namely Scope, Costs and time.

Scope: Which is to illustrates the audience to which the software to be developed

Cost: This means the expenditure or investment made for development process.

Time: This means the timelines for the software development.

\section{RELATED WORKS}

After the era of Internet revolution, most of the standalone application is re-engineered into web application. They are mainly applicable for the large scale transactions, because all of the large scale transaction are having network and their task are fully reduced rather than using software application.

In study, most of the small scale transaction are till dated not re-engineer to web application. The reason behind is accuracy of task performed is high in software application than using web application. Such a scenario, when there is more number of customer are accessing the web application at same time will automatically reduce the task performed and also sometime it results 503i.e, service unavailable messages.

Vulnerability is the major issues of web applications and mainly in large scale transaction due to some coding fault on eBay a fake transaction are made and it lead to huge loss on transaction. Nowadays, many of the software are stopped their product such as visual Basic, struts 1.x and so on. Which mean that, as on feature, if there is any new technology is introduced mean above mentioned type of software are not compatible to that For all above mentioned problem and issues, these paper proposed to implement Goal Question Metrics approach to the small scale 
transaction so that the choice of software development package and migration to various technology can be well determined.

Re-engineering technique is the methodology to provide a better customer satisfaction and adapt the market trends. To prove the importance of re-engineering technique we consider an small scale transaction of a concern and the task performed in that concern by using visual basic v6.0, hence it compatible for their work, the end user support which provide by Microsoft corporation is ended on the year of 2002 as on figure . Even though, the product some feature added with .Net Framework, which is not as much supportive [5].

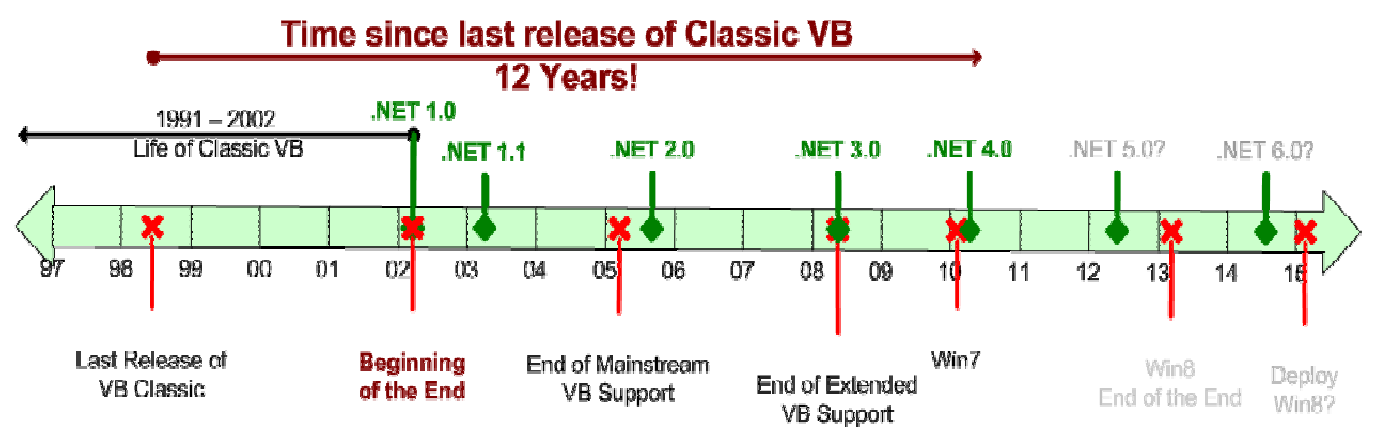

Fig 6: illustrates timeline of Visual Basis6 over Visual Studio

In order to overcome that problem, migration is the technique used by the various vendors. An article describes the Visual Basis v6.0 with Visual Studio migration [5]. By the way of ending the software version will automatically ended the end user support and figure 6 illustrate the timeline of Visual Basis and Visual Studio.

After the introduction of internet, the software development based on web technology is get more effective after 2000 and standalone application are inbuilt with the feature of web integration.

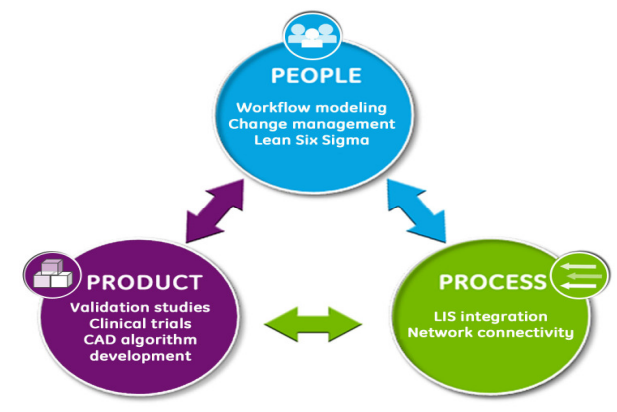

Fig 7: illustrates process flow of SDLC [6]

Let us consider, above scenario as the end of the visual basis v6.0 technology. Because depend upon the people need product are developed and the process of the development product is performed.

\section{PROPOSED APPROACH ON SMALL SCALE TRANSACTION}

In our proposed system, we implemented Goal Question Metrics Approach which is one of the best approaches for re-engineering and Enterprise Resource Planning software development. By 
implementing GQM approach migration upgrading of software will be made effective. An application which developed on basis of standalone will be more flexible and reliable.

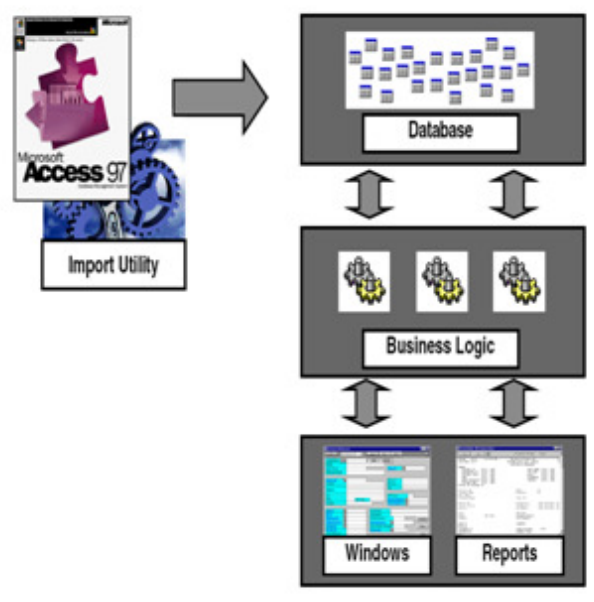

Fig 8: illustrates existing system layout

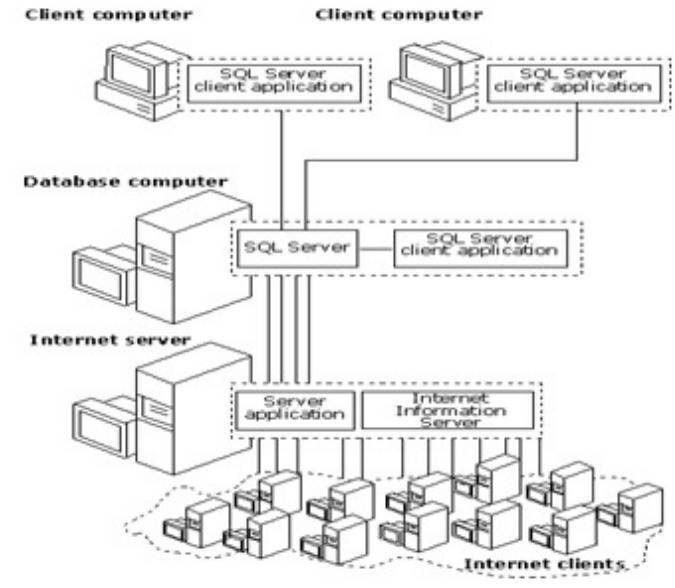

Fig 9: illustrates proposed system layout

The figure 8 illustrates the existing system layout which is developed using Visual Basis v6.0 and back end as Microsoft Access 97. In this system, the backend storage capacity is less and every day they have to make a backup and it has to maintain every day small scale transaction. In order to overcome the problem on proposed system, we implement our proposed approach using Goal Question Metrics and going to implement it Visual Studio because it has the feature capability of web support and web integration [8]. The figure 9 illustrates the architecture of proposed system to overcome for automatic backup capability. In our system, a standalone application is created with web integration and master database is centralized. So every day, after the transaction is over, the proposed system will automatically stored in centralized database and as well as on compact database.

\subsection{The result and discussion on proposed approach}

The Goal Question Metrics Approach which gives clear idea about the Software Development Process. Here, we explain our approach by using real time application. In this system, we developed a Standalone Application and choose .Net Windows Application. The reason for choosing this platform, It support web component and up gradation is more compactable. And for backend, we choose MySql database with remote connectivity enabled and it best suits on mirroring technology [12]. On other end for Mirroring database, we purchase a domain and with support of MySql Database. In Open Source Technology [13], MySql is widely used in major domains and more flexible for all web scripts such as Php, Jsp, and so on. 


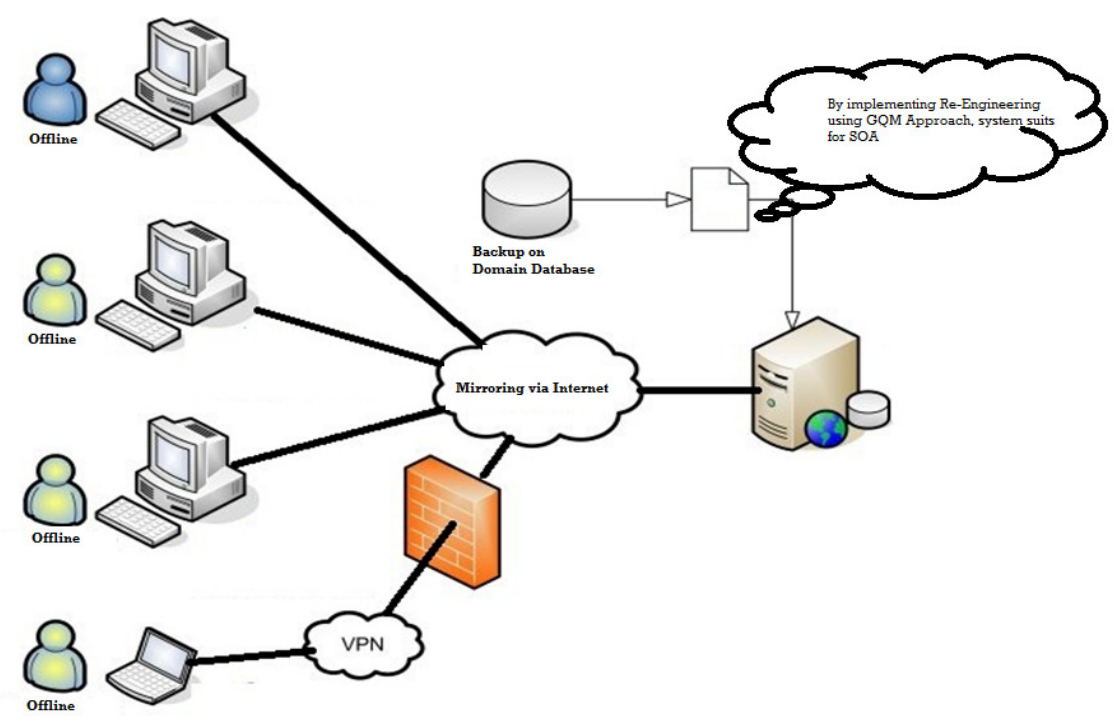

Fig 10- Illustrates implementing methodology using GQM Approach for Re-Engineering

The figure 10 illustrates, the standalone application will store the data in database on client system as offline mode. By enabling remote access technology, when the client system connected to web domain mean auto backup will performed.

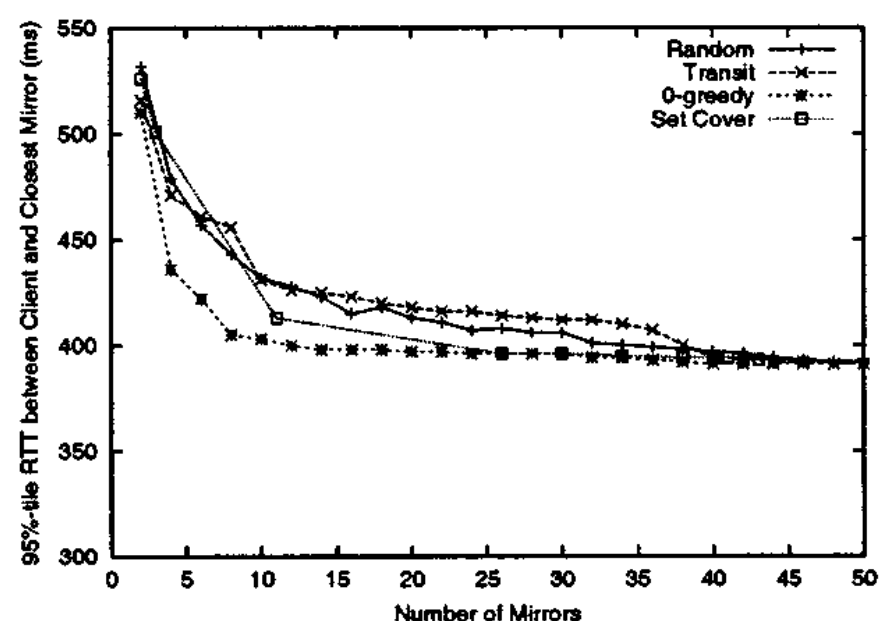

Fig 11- illustrates minimizing Round Trip Time using Apache.

The figure 11 describe implementation of mirroring technique in apache[15] server and proves the proposed system approach on re-engineering is more effective than web technology.

Here, after by fetching all information from the client and that information are stored in domain database. So that, for purpose of monitoring, we can make any type of web scripting for the domain database and can access via internet technology. By way of web scripting, reengineering 
will be made efficient and effective. And GQM Approach [11] makes the software development with well planned question and its metrics. So that, high standard of software development will performed.

\section{CONCLUSION}

By the way of implementing this methodology into Visual Studio, the proposed system will migrated to advanced technology such as web service, embedded hardware devices such as barcode scanner, thumb recognizer, and so on. And hence, we prove that Goal Question Metrics Approach is the best approach for the re-engineering and as well as for including advance technology. So that, instead on re-engineering the standalone application into web application. By the way of automatic backup of data from compact database to centralized database will be efficient and reliable.

\section{REFERENCES}

[1] Aswath Damodaran, Stern School of Business, page(7)Valuation Approaches and Metrics: A Survey of the Theory and Evidence, Nov 2006

[2] Vladimir Mandic, Victor Basili, Lasse Harjuma, Markku Oivo, Jouni Markkula, Page 3-6, Utilizing GQM+Strategies for Business Value Analysis An Approach for Evaluating Business Goals

[3] Frank C. Zagare, Branislav L. Slantchar, page 1-27, Game Theory and Other Modeling Approach

[4] Victor R. Basili, Gianluigi Caldiara, H. Dicter Rombach, page (2-8)The Goal Question Metrics Approach

[5] http://Technet.microsoft.com/en-us/library/cc917543.aspx - explain the reason wit timeline for not providing End user support for standalone applications.

[6] http://www.sdlc.ws/what-is-sdlc/ - explain the basic concepts of Software Development Life cycle.

[7] V. Basilli, J. Kleidrich, M. Lindvall, J. Munch, page (3-11) Bridging the Gap between Strategy and Software development, Information System Strategy and Governance.

[8] Meryem Kassou, Laila kjiri, page(4-6)A Goal Question Metric Approach for Evaluating Security in a Service Oriented Architecture Context, A1-Qualisadi Research \& Development Team, Ecole Nationale Superiecure d'Informatica et d'Analyses de Systemes, ENSIAS, Universite Mohammed VSowisoi.

[9] Victor R.Basili, H. Dicter Rombach, page(2-4), Goal Question Metric Paradigm

[10] Jose Esteves, Joan Pastor, Josep Casanovas, page(4-7) Measuring Sustained Management Support in ERP Implementation Projects: A GQM Approach

[11] Meryem Kassou, Laila Kjiri, page(8-10)A Goal Question Metric Approach for Evaluating Security in a Service Oriented Architecture Context

[12] Eric Cronin, Sugih Jamin, Cheng Jin, Anthony R. Kurc, Danny Raz, page(4-8) Constrained Mirror Placement on the Internet, IEEE JOURNAL ON SELECTED AREAS IN COMMUNICATIONS, VOL. 20, NO. 7, SEPTEMBER 2002

[13 ]Norshidah Mohamed, Muhammad Sadry Abu Seman, Ramlah Hussein, page(1-4) Open Source Software in Information Technology Education, 2009 International Conference on Information Management and Engineering

[14] S“oren Sonnenburg, Mikio L. Braun, Cheng Soon Ong, Samy Bengio, Leon Bottou, Geoffrey Holmes, Yann LeCun, Klaus-Robert M* uller, Fernando Pereira, Carl Edward Rasmussen, page(1923), The Need for Open Source Software in Machine Learning, Journal of Machine Learning Research 8 (2007) 2443-2466, Submitted 7/07; Published 10/07

[15] Eric Cronin, Sugih Jamin, Cheng Jin, Anthony R. Kurc, Danny Raz, page(10-12) Constrained Mirror Placement on the Internet, IEEE JOURNAL ON SELECTED AREAS IN COMMUNICATIONS, VOL. 20, NO. 7, SEPTEMBER 2002 